\title{
Erratum to: A Load-aware Scheme for Providing Heterogeneous Multimedia Broadcast/Multicast Service (Het-MBMS)
}

\author{
Yi-Han Xu • Chee-Onn Chow •
}

Gin-Xian Kok • Mau-Luen Tham

Published online: 18 February 2014

C) Springer Science+Business Media New York 2014

\section{Erratum to: Wireless Pers Commun DOI 10.1007/s11277-014-1628-z}

There is a spelling error in the last author's name in the original publication. The name is correct in this erratum. 